\title{
Bayesian model selection for the latent position cluster model for Social Networks
}

\author{
N. FRIEL ${ }^{1,2}$, C. RYAN ${ }^{1}$ and J. WYSE ${ }^{1,2}$ \\ ${ }^{1}$ School of Mathematical Sciences and ${ }^{2}$ Insight research centre, \\ University College Dublin, Ireland.
}

June 5, 2018

\begin{abstract}
The latent position cluster model is a popular model for the statistical analysis of network data. This approach assumes that there is an underlying latent space in which the actors follow a finite mixture distribution. Moreover, actors which are close in this latent space tend to be tied by an edge. This is an appealing approach since it allows the model to cluster actors which consequently provides the practitioner with useful qualitative information. However, exploring the uncertainty in the number of underlying latent components in the mixture distribution is a very complex task. The current state-of-the-art is to use an approximate form of BIC for this purpose, where an approximation of the log-likelihood is used instead of the true log-likelihood which is unavailable. The main contribution of this paper is to show that through the use of conjugate prior distributions it is possible to analytically integrate out almost all of the model parameters, leaving a posterior distribution which depends on the allocation vector of the mixture model. A consequence of this is that it is possible to carry out posterior inference over the number of components in the latent mixture distribution without using trans-dimensional MCMC algorithms such as reversible jump MCMC. Moreover, our algorithm allows for more reasonable computation times for larger networks than the standard methods using the latentnet package (Krivitsky and Handcock 2008; Krivitsky and Handcock 2013).
\end{abstract}

Key words: collapsed latent position cluster model; reversible jump Markov chain Monte Carlo; Bayesian model choice; social network analysis; finite mixture model

\section{Introduction}

A social network consists of nodes or actors in a graph, for example, individuals or organizations, connected by one or more specific types of interdependency, such as, friendship, business relationships or trade between countries. The analysis of network data has a rich interdisciplinary history finding application in a wide range of areas including sociology (Wasserman and Galaskiewicz 1994), physics (Adamic et al 2001), biology (Michailidis 2012), computer science (Faloutsos et al 1999) and many more. The aims of network analysis are both descriptive and inferential. For example, one might be interested in examining global structure within a network or in analysing network attributes such as the degree distribution as well as the local structure such as the identification of influential or highly connected actors in the network. Inferential goals include hypothesis testing, model comparison and making predictions, for example, how far will a virus spread through a network.

There have been many statistical models proposed for the analysis of network data, the most popular of which include the exponential random graph model see Wasserman and Pattison (1996) and Robins et al (2007) and the stochastic block model of Nowicki and Snijders (2001) and it's variants. For a recent perspective on the statistical analysis of network data, see Kolaczyk (2009). An alternative and popular approach to modelling network data is the latent space approach (Hoff and Handcock 2002). 
Here each actor is embedded in a latent 'social space' in which actors that are close in the latent space are more likely to be tied by an edge. Latent space models (Handcock et al 2007) naturally accommodate many sociological features such as homophily, reciprocity and transitivity. The recent development of Handcock et al (2007) extends the latent space model of Hoff et al (2002) to cluster actors directly, where the positions of actors are assumed to be distributed according to a finite mixture. The latent position cluster model provides a useful interpretation of the network since the underlying latent model provides an automatic means of clustering actors while also providing the uncertainty around the probability of actor membership to each cluster. The R package latentnet (Krivitsky and Handcock 2008; Krivitsky and Handcock 2013), which is part of the statnet suite of packages, can be used to fit the latent position cluster model.

Despite its popularity, a major difficulty with the latent position cluster model is inferring the number of components in the latent mixture distribution. The approach advocated by Handcock et al (2007) is to assess this uncertainty by estimating the Bayesian information criterion (BIC) for each possible model. However, it turns out that it is computationally prohibitive to calculate the maximum log likelihood used in BIC and a tractable approximation is to condition on the minimum Kullback-Leibler estimate of the actors latent positions (Shortreed et al 2006), rather than integrating over the posterior distribution of the actors positions, thereby accounting for the uncertainty in these latent positions. Note that a variational Bayes approximation has been proposed by Salter-Townshend and Murphy (2012) but it too uses the same strategy as (Handcock et al 2007) to infer the number of components. One of the primary contributions of this article is to resolve this issue. To this end we use conjugate prior distributions which allow almost all latent mixture parameters to be integrated out. This results in a collapsed posterior distribution which depends on the vector of allocations of actors to components. The important consequence of this is that the number of components can be inferred without the use of trans-dimensional MCMC techniques such as reversible jump Markov chain Monte Carlo (Richardson and Green 1997). This approach is similar to that presented in Nobile and Fearnside (2007) and Wyse and Friel (2012) for the collapsed finite mixture model and latent block models, respectively. The second important contribution of this paper is that our approach is computationally fast and can be applied to larger networks than is feasible using latentnet. The software which accompanies this paper can be used to implement all the examples presented herein.

The paper begins in Section 2 by describing the latent position cluster model and the current approach to inferring the number of clusters. Section 3 introduces the collapsed form of the model. Cross-model inference is described for the collapsed latent position cluster model in Section 4. Section 5 applies and compares the methodology to current methods for some known social network data. Some discussions follow in Section 6 .

\section{The Latent Position Cluster Model for Social Networks}

Network data may be represented by an $n \times n$ adjacency matrix $\mathbf{Y}=\left\{y_{i j}\right\}_{i, j=1}^{n}$ of binary relations $y_{i j}$ between actors $i$ and $j$, indicating presence or absence of a tie between $i$ and $j$. Ties can be directed where $y_{i j}$ does not necessarily equal to $y_{j i}$ or undirected where $y_{i j}=y_{j i}$ for all $i \neq j$. Self-ties are typically not allowed thus diagonal entries of this matrix take the value 0 . It is also possible to consider networks with integer or weighted values representing the strength of relationship between the two connected actors.

The Latent Position Cluster Model was introduced by Handcock et al (2007) where actor $i$ is assumed to have an unobserved random position, $\mathbf{z}_{i}$, in a $d$-dimensional Euclidean latent social space. The choice of $d=2$ aids visualization but the latent space could be of any dimension with the possibility to infer 
$d$ as a parameter in the model. The probability of a link between two actors is assumed independent of all other links in the network, given the latent locations $\mathbf{Z}=\left\{\mathbf{z}_{i}\right\}_{i=1}^{n}$ of the actors, resulting in the likelihood

$$
L(\mathbf{Y} \mid \mathbf{Z}, \beta)=\prod_{i \neq j} \pi\left(y_{i j} \mid \mathbf{z}_{i}, \mathbf{z}_{j}, \beta\right) .
$$

A logistic regression model is employed, where the probability of a tie between actors $i$ and $j$ depends on the Euclidean distance between $\mathbf{z}_{i}$ and $\mathbf{z}_{j}$ in the latent social space, $\left\|\mathbf{z}_{i}-\mathbf{z}_{j}\right\|$,

$$
\log \left\{\frac{\pi\left(y_{i j}=1 \mid \mathbf{z}_{i}, \mathbf{z}_{j}, \beta\right)}{\pi\left(y_{i j}=0 \mid \mathbf{z}_{i}, \mathbf{z}_{j}, \beta\right)}\right\}=\beta-\left\|\mathbf{z}_{i}-\mathbf{z}_{j}\right\|, \quad \forall i, j \in\{1, \ldots, n\}, i \neq j
$$

where $\beta$ is an intercept parameter. It is assumed that the latent locations $\mathbf{Z}$ are drawn from a finite mixture of $G$ Multivariate Normal components which models the clustering of actors. The mixture model is

$$
\pi\left(\mathbf{Z} \mid \boldsymbol{\mu}, \boldsymbol{\sigma}^{2}, \boldsymbol{\lambda}, G\right)=\prod_{i=1}^{n}\left(\sum_{g=1}^{G} \lambda_{g} f\left(\mathbf{z}_{i} ; \boldsymbol{\mu}_{g}, \sigma_{g}^{2} \mathbf{I}_{d}\right)\right)
$$

where $f\left(\mathbf{z}_{i} ; \boldsymbol{\mu}_{g}, \sigma_{g}^{2} \mathbf{I}_{d}\right)$ is the density function of a Multivariate Normal distribution with cluster means $\boldsymbol{\mu}=\left(\boldsymbol{\mu}_{1}, \ldots, \boldsymbol{\mu}_{G}\right)$ and cluster covariance matrices $\sigma_{g}^{2} \mathbf{I}_{d}$ for $g=1, \ldots, G$, where $\mathbf{I}_{d}$ is the $d$-dimensional identity matrix. The mixing weights are $\boldsymbol{\lambda}=\left(\lambda_{1}, \ldots, \lambda_{G}\right)$, where $\lambda_{g}$ is the probability of actor $i$ belonging to cluster $g \in(1, \ldots, G)$ and $\sum_{g} \lambda_{g}=1$. As usual in mixture modelling, a latent allocation vector $\mathbf{K}=\left(k_{1}, \ldots, k_{n}\right)$ is introduced where $k_{i} \in\{1, \ldots, G\}$ for all $i=1, \ldots, n$. If actor $i$ belongs to cluster $g$ then $k_{i}=g$. This provides a tractable augmented expression for the mixture model,

$$
\pi\left(\mathbf{Z}, \mathbf{K} \mid \boldsymbol{\mu}, \boldsymbol{\sigma}^{2}, \boldsymbol{\lambda}, G\right)=\prod_{i=1}^{n} \prod_{g=1}^{G}\left(\lambda_{g} f\left(\mathbf{z}_{i} ; \boldsymbol{\mu}_{g}, \sigma_{g}^{2} \mathbf{I}_{d}\right)\right)^{\mathbb{1}\left(k_{i}=g\right)},
$$

where the indicator function $\mathbb{1}\left(k_{i}=g\right)$ is 1 if $k_{i}=g$ or 0 otherwise. This joint density of $\mathbf{Z}$ and $\mathbf{K}$ can be factorised as

$$
\pi\left(\mathbf{Z} \mid \mathbf{K}, \boldsymbol{\mu}, \boldsymbol{\sigma}^{2}, G\right) \pi(\mathbf{K} \mid \lambda, G)=\prod_{i=1}^{n} \prod_{g=1}^{G} f\left(\mathbf{z}_{i} ; \boldsymbol{\mu}_{g}, \sigma_{g}^{2} \mathbf{I}_{d}\right)^{\mathbb{1}\left(k_{i}=g\right)} \prod_{g=1}^{G} \lambda_{g}^{\left(\sum_{i=1}^{n} \mathbb{1}\left(k_{i}=g\right)\right)} .
$$

The posterior distribution can be factorised as

$$
\pi\left(\mathbf{Z}, \boldsymbol{\mu}, \boldsymbol{\sigma}^{2}, \boldsymbol{\lambda}, \beta, \mathbf{K}, G \mid \mathbf{Y}\right) \propto L(\mathbf{Y} \mid \mathbf{Z}, \beta) \pi\left(\mathbf{Z} \mid \boldsymbol{\mu}, \boldsymbol{\sigma}^{2}, \mathbf{K}, G\right) \pi(\mathbf{K} \mid \boldsymbol{\lambda}, G) \pi(\boldsymbol{\lambda} \mid G) \pi\left(\boldsymbol{\mu} \mid \boldsymbol{\sigma}^{2}, G\right) \pi\left(\boldsymbol{\sigma}^{2} \mid G\right) \pi(\beta) \pi(G) .
$$

The likelihood as defined by equations (1) and (2) involves the product of all possible $n \times(n-1)$ pairs of actors. Raftery et al (2012) adopt the epidemiological approach of case-control sampling (Breslow 1996) to approximate the likelihood thus reducing computation from $O\left(n^{2}\right)$ to $O(n)$. Our analysis does not approximate the likelihood. Instead computation time is reduced by integrating out the clustering parameters, $\boldsymbol{\theta}=\left(\boldsymbol{\mu}, \boldsymbol{\sigma}^{2}, \boldsymbol{\lambda}\right)$.

Prior distributions on the model parameters $\beta, \boldsymbol{\mu}, \boldsymbol{\tau}=\frac{1}{\boldsymbol{\sigma}^{2}}$ and $\boldsymbol{\lambda}$ are

$$
\begin{aligned}
& \beta \sim \operatorname{Normal}(\xi, \psi), \boldsymbol{\mu}_{g} \mid \tau_{g} \sim \operatorname{MVN}_{d}\left(0, \frac{\omega^{2}}{\tau_{g}} \mathbf{I}_{d}\right), \\
& \tau_{g}=1 / \sigma_{g}^{2} \sim \operatorname{Gamma}\left(\frac{\alpha}{2}, \frac{\delta}{2}\right), \quad \boldsymbol{\lambda} \sim \operatorname{Dirichlet}(\boldsymbol{\nu}),
\end{aligned}
$$


where the prior hyper-parameters $\phi=\left(\xi, \psi, \alpha, \delta, \boldsymbol{\nu}, \omega^{2}\right)$ are user specified. The priors model dependency on the latent positions and their hyperparameter values require careful choice. See a discussion of this point in the rejoinder to the article (Handcock et al 2007). Following Handcock et al (2007), $\xi=0$ and $\psi=2$ allowing a wide range of values for $\beta$. The prior hyper-parameters for the mixing weights $\boldsymbol{\lambda}$ are fixed as in Handcock et al (2007), where $\nu_{g}=3$ for $g=1, \ldots, G$ to put low probability on small group sizes. The prior on the number of clusters is Poisson(1) distributed following Nobile and Fearnside (2007) which penalizes the addition of empty groups. Handcock et al (2007) and Richardson \& Green (1997) instead employ a Uniform prior distribution between 1 and a pre-specified integer $G$. However, in a technical report, Nobile (2007) argues that there is a significant effect on the posterior distribution of $G$ from models with empty components. The use of a Poisson prior reduces this effect, and has been used by other authors, including Phillips and Smith (1996) and Stephens (2000). The conditioning of $\boldsymbol{\mu}_{g}$ on $\tau_{g}$ is a fully conjugate prior and is commonly employed in the literature (Nobile and Fearnside 2007; Dellaportas and Papageorgiou 2006). We use this to allow both parameters to be integrated out of the model as in Nobile and Fearnside (2007). This differs from the original specification of the model by Handcock et al (2007), where the prior distribution for the cluster means is

$$
\boldsymbol{\mu}_{g} \sim \operatorname{MVN}_{d}\left(\mathbf{0}, \omega^{2} \mathbf{I}_{\mathbf{d}}\right),
$$

which does not depend on the cluster variances.

\subsection{Choosing the number of clusters for the latent position cluster model}

The model evidence (sometimes called the marginal or integrated likelihood) plays a central role in the Bayesian approach to model choice. The model evidence for the latent position cluster model with clustering parameters denoted $\boldsymbol{\theta}=\left(\boldsymbol{\mu}, \boldsymbol{\sigma}^{2}, \boldsymbol{\lambda}\right)$ and all terms conditional on $G$ is given by

$$
\begin{aligned}
\pi(\mathbf{Y} \mid G) & =\int_{\mathbf{Z}} \int_{\boldsymbol{\theta}} \int_{\beta} \pi(\mathbf{Y}, \mathbf{Z} \mid \beta, \boldsymbol{\theta}) \pi(\beta) \pi(\boldsymbol{\theta}) d \beta d \boldsymbol{\theta} d \mathbf{Z} \\
& =\int_{\mathbf{Z}} \int_{\boldsymbol{\theta}} \int_{\beta} L(\mathbf{Y} \mid \mathbf{Z}, \beta) \pi(\mathbf{Z} \mid \boldsymbol{\theta}) \pi(\beta) \pi(\boldsymbol{\theta}) d \beta d \boldsymbol{\theta} d \mathbf{Z} .
\end{aligned}
$$

This quantity represents the probability of the observed data given a latent mixture model with $G$ components. Here the variable $G$ can be interpreted as a model index.

Using the model evidence, Bayes theorem can be used to evaluate the posterior probability, $\pi(G \mid \mathbf{Y}) \propto$ $\pi(\mathbf{Y} \mid G) \pi(G)$. See Friel and Wyse (2012) for a recent review of model evidence estimation. However, integration across all possible values of $\mathbf{Z}$ in equation (4) is intractable, due to the dimensionality of Z. A pragmatic approach taken by Handcock et al (2007) is to condition on a fixed estimate of latent actor locations $\hat{\mathbf{Z}}$. These are estimated using minimum Kullback-Leibler position estimation (Shortreed et al 2006). Since the logistic regression model is a function of distances between actors rather than the actual latent positions, the estimate $\hat{\mathbf{Z}}$ is found by minimizing the Kullback-Leibler divergence between the true unknown model distances and the MCMC sample position based distances. See Appendix A of Handcock et al (2007) for further details. The model evidence is approximated as

$$
\begin{aligned}
\pi(\mathbf{Y} \mid G) \approx \pi(\mathbf{Y}, \hat{\mathbf{Z}} \mid G) & =\int_{\boldsymbol{\theta}} \int_{\beta} \pi(\mathbf{Y} \mid \hat{\mathbf{Z}}, \beta) \pi(\hat{\mathbf{Z}} \mid \boldsymbol{\theta}) \pi(\beta) \pi(\boldsymbol{\theta}) d \beta d \boldsymbol{\theta} \\
& =\int_{\beta} \pi(\mathbf{Y} \mid \hat{\mathbf{Z}}, \beta) \pi(\beta) d \beta \int_{\boldsymbol{\theta}} \pi(\hat{\mathbf{Z}} \mid \boldsymbol{\theta}) \pi(\boldsymbol{\theta}) d \boldsymbol{\theta}
\end{aligned}
$$


The 'best' $G$ component model corresponds to the largest value of $\pi(\mathbf{Y}, \hat{\mathbf{Z}} \mid G)$. This approach does not take the uncertainty of $\mathbf{Z}$ into account, moreover it is unclear how this approximation impacts upon the assessment of the number of latent mixture components. The BIC approximation to the model evidence (Schwarz 1978) is employed by Handcock at al (2007) to approximate the integrals in equation (5). The first integral is estimated by a BIC type approximation denoted by $\mathrm{BIC}_{l r}$ for logistic regression,

$$
\log \left\{\int_{\beta} \pi(\mathbf{Y} \mid \hat{\mathbf{Z}}, \beta) \pi(\beta) d \beta\right\} \approx \frac{1}{2} \mathrm{BIC}_{l r}=\frac{1}{2}\left(2 \log \{\pi(\mathbf{Y} \mid \hat{\mathbf{Z}}, \hat{\beta}(\hat{\mathbf{Z}}))\}-d_{l r} \log \left\{n_{l r}\right\}\right)
$$

where $\hat{\beta}(\hat{\mathbf{Z}})$ is the maximum likelihood estimator of $\beta$ given a fixed posterior estimate of the latent locations $\hat{\mathbf{Z}}, n_{l r}$ is the number of ties in the network and $d_{l r}$ is the dimension of $\beta$, the number of parameters in the logistic regression model. Actor covariate data may be included in the logistic regression model (Handcock et al (2007)), in which case $d_{l r}>1$. The second integral in equation (5) is approximated using a similar $\mathrm{BIC}$ approximation denoted $\mathrm{BIC}_{l p}$ for the latent positions,

$$
\left.\log \left\{\int_{\boldsymbol{\theta}} \pi(\hat{\mathbf{Z}} \mid \boldsymbol{\theta}) \pi(\boldsymbol{\theta}) d \boldsymbol{\theta}\right\} \approx \frac{1}{2} \mathrm{BIC}_{l p}=\frac{1}{2}\left(2 \log \{\pi(\hat{\mathbf{Z}} \mid \hat{\boldsymbol{\theta}}(\hat{\mathbf{Z}}))\}-d_{l p} \log \{n\}\right)\right),
$$

where $\hat{\boldsymbol{\theta}}(\hat{\mathbf{Z}})$ is the maximum likelihood estimator of $\boldsymbol{\theta}$ given the fixed posterior estimate of the latent positions $\hat{\mathbf{Z}}$ and $d_{l p}$ is the number of parameters in the mixture model.

A similar approach is carried out in the variational Bayesian framework (Salter-Townshend and Murphy 2012) where $\hat{\mathbf{Z}}$ is the modal variational posterior estimate of the latent positions. This paper avoids the approximations of equations (5), (6) and (7) by modelling jointly, the number of components $G$ in a fully probabilistic Bayesian approach as well as exploring uncertainty in $\mathbf{Z}$ and $\beta$ using MCMC methods. This is made computationally feasible by collapsing or integrating out the clustering parameters from the model analytically (Section 3). Thus the marginal probability $\pi(G \mid \mathbf{Y}$ ) can be estimated directly via MCMC sampling of the collapsed posterior.

\section{Collapsing the Model}

It is possible to integrate out or collapse the clustering parameters $\boldsymbol{\theta}$ from the posterior distribution analytically by using the conjugate priors described in Section 2. This yields a collapsed posterior 
distribution for the latent position cluster model,

$$
\begin{aligned}
\pi(\mathbf{Z}, \beta, \mathbf{K}, G \mid \mathbf{Y}) \propto & \int_{\boldsymbol{\lambda}} \int_{\boldsymbol{\sigma}} \int_{\boldsymbol{\mu}} L(\mathbf{Y} \mid \mathbf{Z}, \beta) \pi\left(\mathbf{Z} \mid \boldsymbol{\mu}, \boldsymbol{\sigma}^{2}, \mathbf{K}, G\right) \pi\left(\boldsymbol{\mu} \mid \boldsymbol{\sigma}^{2}, G\right) \pi\left(\boldsymbol{\sigma}^{2} \mid G\right) \pi(\mathbf{K} \mid \boldsymbol{\lambda}, G) \\
= & L(\mathbf{Y} \mid \mathbf{Z}, \beta) \pi(\mathbf{Z} \mid \mathbf{K}, G) \pi(\mathbf{K} \mid G) \pi(\beta) \pi(G) \\
= & \prod_{i=1}^{n} \prod_{j \neq i} \frac{\exp \left\{y_{i j}\left(\beta-\| \mathbf{z}_{i}-\mathbf{z}_{j}||\right)\right\}}{1+\exp \left\{\beta-\| \mathbf{z}_{i}-\mathbf{z}_{j}||\right\}} \\
& \times \prod_{g=1}^{G}\left(\frac{\Gamma\left(\frac{n_{g} d+\alpha}{2}\right)}{\left(n_{g}+\frac{1}{\omega^{2}}\right)^{\frac{d}{2}}}\left(\delta+\sum_{i: k_{i}=g}\left\|z_{i}\right\|^{2}-\frac{\left\|\sum_{i: k_{i}=g} z_{i}\right\|^{2}}{\left(n_{g}+\frac{1}{\omega^{2}}\right)}\right)^{\left.-\left(\frac{n_{g} d+\alpha}{2}\right)\right)}\right) \\
& \times \frac{\Gamma(G \nu)}{\Gamma(\nu)^{G}} \pi^{-\frac{d n}{2}} \frac{(\delta)^{\frac{G \alpha}{2}}}{\Gamma\left(\frac{\alpha}{2}\right)^{G}}\left(\omega^{2}\right)^{-\frac{G d}{2}} \frac{\prod_{g=1}^{G} \Gamma\left(n_{g}+\nu\right)}{\Gamma(n+G \nu)} \\
& \times \frac{1}{\sqrt{2 \pi \psi}} \exp \left\{-\frac{(\beta-\xi)^{2}}{2 \psi}\right\} \times \frac{\exp \{-1\}}{G !},
\end{aligned}
$$

where $n_{g}=\sum_{i=1}^{n} \mathbb{1}\left(k_{i}=g\right)$. Full details of the integration is given in the Appendix.

This is similar to the approach of Nobile and Fearnside (2007) and Wyse and Friel (2012), where the allocation sampling algorithm was developed for the collapsed finite mixture model and latent block models, respectively. This paper extends the approach to the latent position cluster model for social networks, where the latent actor locations are analogous to the observed data in Nobile and Fearnside (2007). The collapsed posterior for the latent position cluster model (equation 8) depends on $\mathbf{Z}$ and $\mathbf{K}$, the latent positions and the allocation vector, respectively. In particular, $\mathbf{K}$ is of fixed dimension, but crucially, it provides information on the number of components in the model. In this way, it is possible to carry out trans-model inference over a fixed dimensional parameter space, unlike reversible jump MCMC (Richardson and Green 1997) for the full model which involves algorithmic moves of variable dimension. Additionally, the collapsed posterior involves a much reduced parameter space, since the component means, variance and mixing weights are analytically integrated out. The advantage of our approach is improved computational efficiency, reduced parameter storage requirements and a reduction in variability due to the removal of the uncertainty associated with the clustering parameters which have been integrated out of the model.

\section{A trans-model algorithm for the collapsed latent position cluster model}

Markov chain Monte Carlo sampling of the collapsed posterior distribution for the latent position cluster model is carried out using a Metropolis-within-Gibbs algorithm. As full-conditional distributions for the positions $\mathbf{Z}$ and intercept $\beta$ are not of standard form, Metropolis-Hastings-within-Gibbs updates are required. A standard Gibbs update is carried out to update the allocation vector $\mathbf{K}$ and we suggest a further 3 Metropolis-Hastings-within-Gibbs moves to update $\mathbf{K}$ without changing the number of components in the model. Finally, a trans-model ejection/absorption move proposes the addition or removal of a component, changing only the fixed dimensional allocation vector $\mathbf{K}$. The moves are similar to the approach of Nobile and Fearnside (2007) and Wyse and Friel (2012). 


\subsection{Metropolis-Hastings-within-Gibbs update for the latent positions $\mathrm{Z}$}

Metropolis-Hastings-within-Gibbs sampling is carried out using the collapsed full-conditional distribution for the positions $\mathbf{Z}$,

$$
\begin{aligned}
\pi(\mathbf{Z} \mid \mathbf{K}, \beta, G, \mathbf{Y}) \propto & L(\mathbf{Y} \mid \mathbf{Z}, \beta) \pi(\mathbf{Z} \mid \mathbf{K}, G) \\
\propto & \prod_{i=1}^{n} \prod_{j \neq i} \frac{\exp \left\{y_{i j}\left(\beta-\left\|\mathbf{z}_{i}-\mathbf{z}_{j}\right\|\right)\right\}}{1+\exp \left\{\beta-\left\|\mathbf{z}_{i}-\mathbf{z}_{j}\right\|\right\}} \\
& \times \prod_{g=1}^{G}\left(\frac{\Gamma\left(\frac{n_{g} d+\alpha}{2}\right)}{\left(n_{g}+\frac{1}{\omega^{2}}\right)^{\frac{d}{2}}}\left(\delta+\sum_{i: k_{i}=g}\left\|\mathbf{z}_{i}\right\|^{2}-\frac{\left\|\sum_{i: k_{i}=g} \mathbf{z}_{i}\right\|^{2}}{\left(n_{g}+\frac{1}{\omega^{2}}\right)}\right)^{-\left(\frac{n_{g} d+\alpha}{2}\right)}\right) .
\end{aligned}
$$

A full sweep consists of visiting each $\mathbf{z}_{i}$ for $i=1, \ldots, n$ and proposing an update of $\mathbf{z}_{i}$ which is accepted or rejected using the usual Metropolis-Hastings accept/reject probability, as outlined in Update (1).

Update 1: Metropolis Hastings update of the actor locations $\mathbf{Z}$

1 At iteration $t$;

2 for $i=1, \ldots, n$ do

$3 \quad$ Propose $\mathbf{z}_{i}^{\prime} \sim q\left(\mathbf{z}_{i}^{t} \rightarrow \mathbf{z}_{i}^{\prime}\right)$, where the proposal distribution $q$ is a Multivariate Normal distribution $f\left(\mathbf{z}_{i}^{\prime} ; \mathbf{z}_{i}^{t}, \sigma_{\mathbf{Z}}^{2} \mathbf{I}_{d}\right)$, with mean $\mathbf{z}_{i}^{t}$ and covariance matrix $\sigma_{\mathbf{Z}}^{2} \mathbf{I}_{d}$;

$4 \quad$ Accept $\mathbf{z}_{i}^{t+1}=\mathbf{z}_{i}^{\prime}$ with probability $\min (1, \alpha)$ where,

$$
\alpha=\frac{\pi\left(\mathbf{Z}^{\prime} \mid \mathbf{K}, \beta, G, \mathbf{Y}\right)}{\pi\left(\mathbf{Z}^{t} \mid \mathbf{K}, \beta, G, \mathbf{Y}\right)} \frac{q\left(\mathbf{z}_{i}^{\prime} \rightarrow \mathbf{z}_{i}^{t}\right)}{q\left(\mathbf{z}_{i}^{t} \rightarrow \mathbf{z}_{i}^{\prime}\right)}
$$

5 end

Otherwise set $\mathbf{z}_{i}^{t+1}=\mathbf{z}_{i}^{t}$.

\subsection{Metropolis-Hastings-within-Gibbs update for the intercept $\beta$}

The collapsed full-conditional distribution for the intercept parameter $\beta$ is not of standard form,

$$
\begin{aligned}
\pi(\beta \mid \mathbf{Z}, \mathbf{Y}) & \propto \pi(\mathbf{Y} \mid \mathbf{Z}, \beta) \pi(\beta) \\
& \propto \prod_{i=1}^{n} \prod_{j \neq i}\left(\frac{\exp \left\{y_{i j}\left(\beta-\left\|\mathbf{z}_{i}-\mathbf{z}_{j}\right\|\right)\right\}}{1+\exp \left\{\beta-\left\|\mathbf{z}_{i}-\mathbf{z}_{j}\right\|\right\}}\right) \exp \left\{-\frac{(\beta-\xi)^{2}}{2 \psi}\right\} .
\end{aligned}
$$


Thus the Metropolis-Hastings-within-Gibbs Update (2) is executed.

Update 2: Metropolis Hastings update of the intercept parameter $\beta$

1 At iteration $t$;

2 Propose $\beta^{\prime} \sim q\left(\beta^{t} \rightarrow \beta^{\prime}\right)$, where the proposal distribution $q$ is a Normal distribution $f\left(\beta^{\prime} ; \beta^{t}, \sigma_{\beta}^{2}\right)$, with mean $\beta^{t}$ and variance $\sigma_{\beta}^{2}$;

3 Accept $\beta^{t+1}=\beta^{\prime}$ with probability $\min (1, \alpha)$ where,

$$
\alpha=\frac{\pi\left(\beta^{\prime} \mid \mathbf{Z}, \mathbf{Y}\right)}{\pi\left(\beta_{t} \mid \mathbf{Z}, \mathbf{Y}\right)} \frac{q\left(\beta^{\prime} \rightarrow \beta^{t}\right)}{q\left(\beta^{t} \rightarrow \beta^{\prime}\right)} ;
$$

4 Otherwise set $\beta^{t+1}=\beta^{t}$.

\subsection{Moves to update the cluster membership vector $\mathrm{K}$}

\subsubsection{Gibbs update}

To update the allocation vector $\mathbf{K}$, a standard Gibbs update is performed using its full-conditional distribution,

$$
\begin{aligned}
\pi(\mathbf{K} \mid \mathbf{Z}, G, \phi) & \propto \pi(\mathbf{Z} \mid \mathbf{K}, G, \phi) \pi(\mathbf{K} \mid G, \phi) \\
& \propto \prod_{g=1}^{G}\left(\frac{\Gamma\left(\frac{n_{g} d+\alpha}{2}\right)}{\left(n_{g}+\frac{1}{\omega^{2}}\right)^{\frac{d}{2}}}\left(\delta+\sum_{i: k_{i}=g}\left\|\mathbf{z}_{i}\right\|^{2}-\frac{\left\|\sum_{i: k_{i}=g} \mathbf{z}_{i}\right\|^{2}}{\left(n_{g}+\frac{1}{\omega^{2}}\right)}\right)^{-\left(\frac{n_{g} d+\alpha}{2}\right)}\right) \Gamma\left(n_{g}+\nu\right) .
\end{aligned}
$$

A full sweep consists of visiting each $k_{i}$ for $i=1, \ldots, n$ and carrying out Update 3 .



A further 3 moves are proposed to update $\mathbf{K}$, without changing the number of components in the model. These mimic the allocation sampling algorithm of Nobile and Fearnside (2007) and serve to update several actor allocations simultaneously, searching more easily across the discrete set of possible allocation vectors.

\subsubsection{Move 1}

The first Metropolis-Hastings move to update $\mathbf{K}$ without changing the number of groups reallocates the actors of two components $j_{1}$ and $j_{2}$ selected at random from the $G$ available groups. Observations in both groups are re-allocated to component $j_{1}$ with probability $p$ and to component $j_{2}$ with probability 
$1-p$ where $p$ is $\operatorname{Beta}(1,1)$ distributed. The current and proposed allocation vectors are $\mathbf{K}$ and $\mathbf{K}^{\prime}$ respectively and the proposal is symmetric. The move is accepted with probability $\min (1, \alpha)$ where,

$$
\alpha=\frac{\pi\left(\mathbf{Z} \mid G, \mathbf{K}^{\prime}, \phi\right)}{\pi(\mathbf{Z} \mid G, \mathbf{K}, \phi)} .
$$

\subsubsection{Move 2}

The second Metropolis-Hastings update proposes to move a subset of members of one component at random to another component. The idea is that, if these observations are already grouped together into one component, then they may be similar in nature. Thus it may be possible to move them together at the same time to another component. Components $j_{1}$ and $j_{2}$ are randomly selected among the $G$ available groups. If $n_{j_{1}} \neq 0, m$ random observations are selected from component $j_{1}$ and proposed to move to component $j_{2}$, where $m$ is drawn from a Uniform distribution on $\left\{1, \ldots, n_{j_{1}}\right\}$. The proposal is accepted with probability $\min (1, \alpha)$ where,

$$
\alpha=\frac{\pi\left(\mathbf{Z} \mid G, \mathbf{K}^{\prime}, \phi\right)}{\pi(\mathbf{Z} \mid G, \mathbf{K}, \phi)} \frac{q\left(\mathbf{K}^{\prime} \rightarrow \mathbf{K}\right)}{q\left(\mathbf{K} \rightarrow \mathbf{K}^{\prime}\right)}
$$

and where,

$$
\frac{q\left(\mathbf{K}^{\prime} \rightarrow \mathbf{K}\right)}{q\left(\mathbf{K} \rightarrow \mathbf{K}^{\prime}\right)}=\frac{n_{j_{1}}}{n_{j_{2}}+m} \frac{n_{j_{1}} ! n_{j_{2}} !}{\left(n_{j_{1}}-m\right) !\left(n_{j_{2}}+m\right) !}
$$

\subsubsection{Move 3}

The third proposal is similar to the first. Again, the actors of randomly selected components $j_{1}$ and $j_{2}$ are allocated to one of the two groups. However, the probability $p$ is no longer constant for all actors. Instead, in a random sequence, actor $i$ is proposed to move with probability $p_{j}^{(i)}$ for $j \in\left\{j_{1}, j_{2}\right\}$, where $p_{j}^{(i)}$ is proportional to the probability that component $j$ generated the $i$-th observation, conditional on its value $\mathbf{z}_{i}$, on the previously re-allocated observations and on their new allocations. See Appendix $A 2$ of Nobile and Fearnside (2007) for further details. The proposal is accepted with probability $\min (1, \alpha)$ where,

$$
\alpha=\frac{\pi\left(\mathbf{Z} \mid G, \mathbf{K}^{\prime}, \phi\right)}{\pi(\mathbf{Z} \mid G, \mathbf{K}, \phi)} \frac{q\left(\mathbf{K}^{\prime} \rightarrow \mathbf{K}\right)}{q\left(\mathbf{K} \rightarrow \mathbf{K}^{\prime}\right)}
$$

and where,

$$
\frac{q\left(\mathbf{K}^{\prime} \rightarrow \mathbf{K}\right)}{q\left(\mathbf{K} \rightarrow \mathbf{K}^{\prime}\right)}=\prod_{i} \frac{p_{k_{i}}^{(i)}}{p_{k_{i}^{\prime}}^{(i)}}
$$

\subsubsection{Absorption/ejection moves}

This pair of trans-model Metropolis-Hastings-within-Gibbs moves involve the addition or removal of a component. To add a component to the model, a new component $j_{2}^{\prime}$ is ejected from a randomly selected existing component $j_{1}$. The members of component $j_{1}$ are allocated to component $j_{1}^{\prime}$ with probability $p$ and to component $j_{2}^{\prime}$ with probability $1-p$, where $p$ is $\operatorname{Beta}(a, a)$ distributed. In the reverse move, one component absorbs another. The absorption/ejection moves do not change the dimension of the parameter space since $\mathbf{K}$ is of fixed dimension. 
Suppose an absorption or ejection move is attempted from current state $\{\mathbf{K}, G\}$ to $\left\{\mathbf{K}^{\prime}, G^{\prime}\right\}$, where where $G$ and $G^{\prime}$ are the current and proposed number of groups in the model respectively. The probability of choosing a split move is $p^{e}=(1,0.5, \ldots, 0.5,0)$, where $p_{1}^{e}=1$ since a 1 component model must be split and $p_{G_{\max }}^{e}=0$ as it is not possible to split a $G_{\max }$ component model, where $G_{\max }$ is the maximum number of components allowed. This is a user specified value taken to be $\lfloor n / 2\rfloor$ in our examples.

The move is accepted with probability $\min (1, \alpha)$ where,

$$
\alpha=\frac{\pi\left(\mathbf{Z}^{\prime} \mid \mathbf{K}^{\prime}, G^{\prime}, \phi\right) \pi\left(\mathbf{K}^{\prime} \mid G^{\prime}, \phi\right) \pi\left(G^{\prime}\right)}{\pi(\mathbf{Z} \mid \mathbf{K}, G, \phi) \pi(\mathbf{K} \mid G, \phi) \pi(G)} \frac{q\left(\left\{\mathbf{K}^{\prime}, G^{\prime}\right\} \rightarrow\{\mathbf{K}, G\}\right)}{q\left(\{\mathbf{K}, G\} \rightarrow\left\{\mathbf{K}^{\prime}, G^{\prime}\right\}\right)},
$$

and where,

$$
\frac{q\left(\left\{\mathbf{K}^{\prime}, G^{\prime}\right\} \rightarrow\{\mathbf{K}, G\}\right)}{q\left(\{\mathbf{K}, G\} \rightarrow\left\{\mathbf{K}^{\prime}, G^{\prime}\right\}\right)}=\frac{1-p_{G+1}^{e}}{p_{G}^{e}} \frac{2 \Gamma(a)}{\Gamma(2 a)} \frac{\Gamma\left(2 a+n_{j 1}\right)}{\Gamma\left(a+n_{j_{1}^{\prime}}\right) \Gamma\left(a+n_{j_{2}^{\prime}}\right)} .
$$

The reverse absorb move is analogous with the probability of proposing an absorb move, $p^{a}=(0,0.5, \ldots, 0.5,1)$ and acceptance probability $\min \left(1, \alpha^{-1}\right)$.

\subsection{The collapsed sampling algorithm}

To sample from the collapsed latent position cluster model, we implement Algorithm (4). The output of the Markov chain at iteration $t$ is denoted by $\left(\mathbf{Z}^{(t)}, \beta^{(t)}, \mathbf{K}^{(t)}\right)$.

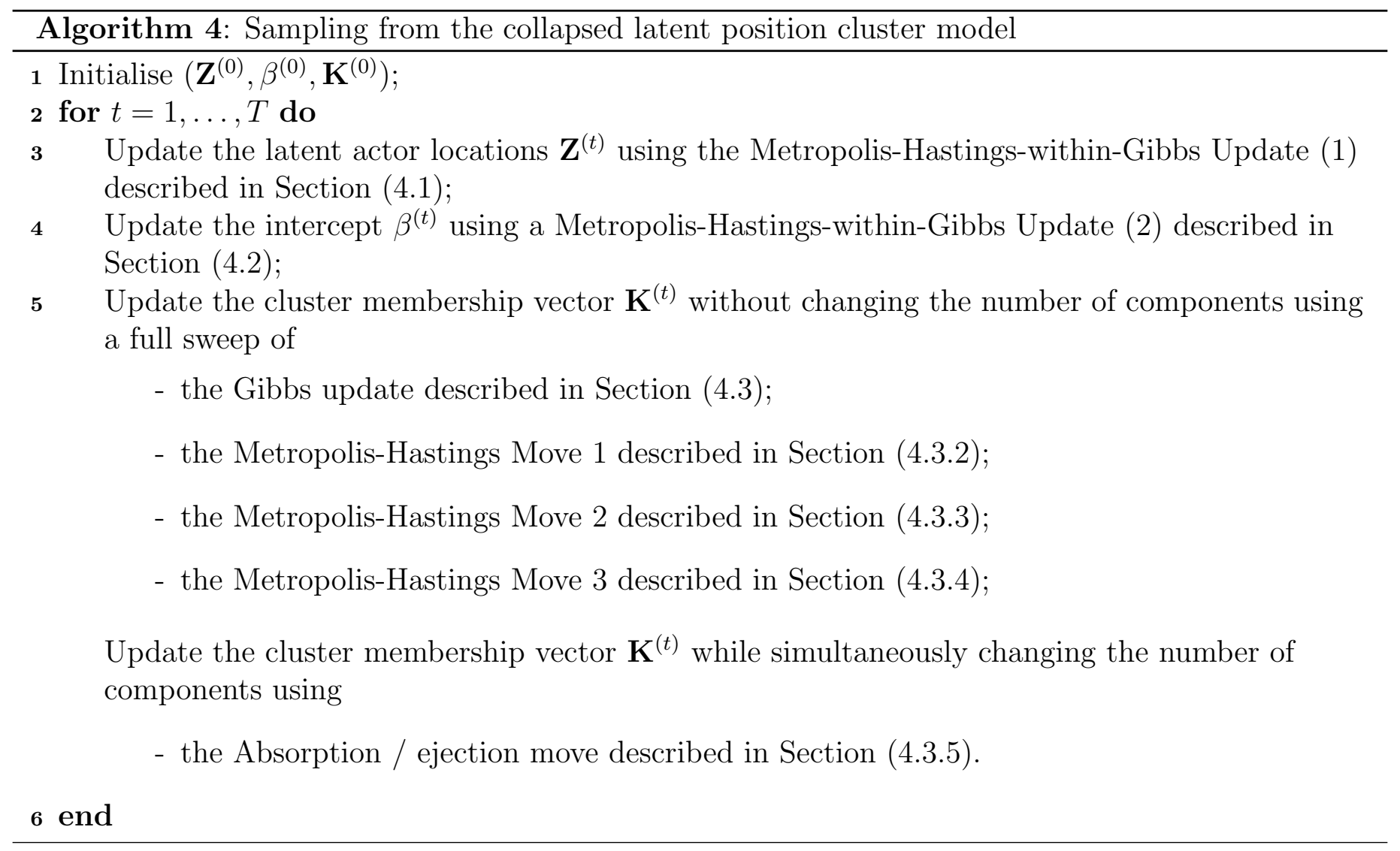




\subsection{Post-processing}

Post processing is required due to the invariance of the likelihood to reflections, rotations and translations of the latent space and to the re-labelling of clusters. This is due to the fact that the actor positions appear in the likelihood as function of Euclidean distance only. To address this problem, a Procrustes transformation (Sibson 1978) is used to match each iteration to a reference configuration. The realisation of the Markov chain with the highest likelihood value is used as a reference configuration. The likelihood invariance to the switching of cluster labels is corrected by iteratively minimising the cost associated with all possible label permutations using the square assignment algorithm of Carpeneto and Toth (1980) as in Nobile and Fearnside (2007) and Wyse and Friel (2012).

\section{Results}

The methods are illustrated using some well known social networks, Sampson's 18 node network (Sampson 1968), Zachary's 34 node karate club network (Zachary 1977) and a 62 node network of New Zealand Dolphins (Lusseau et al 2003). The examples serve to illustrate our methodology, to highlight the importance of model uncertainty for well known social networks and to make comparisons with inference using latentnet (Krivitsky and Handcock 2008; Krivitsky and Handcock 2013) and the variational approximation to the posterior using VBLPCM.

Inference using latentnet involves sampling from the full posterior of Handcock et al (2007). The number of clusters $G$ is fixed and inference is carried out separately for $G=1, \ldots, G_{\max }$. The BIC approximation to the model evidence is used to choose the 'best' model.

The Variational Bayes approach to inference is implemented using VBLPCM (Salter-Townshend and Murphy 2012). The Kullback-Leibler divergence from an approximate fully factorised variational posterior to the true posterior distribution is minimized. Inference is carried out separately for $G=$ $1, \ldots, G_{\max }$ component models. A good initialisation of the variational parameters is important (SalterTownshend and Murphy 2012). The Fruchterman-Reingold layout is used to initialise the latent positions, followed by the use of mclust (Fraley and Raftery 2002; Fraley and Raftery 2003) to initialise the clustering parameters. The Fruchterman-Reingold layout algorithm is itself initialised using a random configuration, thus different results will be found each time. The variational approximation which is 'closest' to the true posterior in terms of Kullback-Leibler divergence is chosen as the best $G$ component model from 10 different initialisations. The BIC approximation to the model evidence is then used to choose the number of components (Section 2.1).

\subsection{Sampson's monks}

Sampson (1968) conducted a social science study of 18 monks in a monastery during the time of Vatican II. During the study, a political 'crisis in the cloister' resulted in the expulsion of four monks and the voluntary departure of several others. A directed network was recorded where each monk was asked to rank 3 friends across 3 points in time. For the purposes of illustrating our methodology, we use the aggregated version of this network widely used in social network analysis literature. However the extension of the latent position cluster model to temporal networks is an open problem.

A 3 or 4 component model is widely accepted as the most suitable clustering for this data. The collapsed model inference was in agreement, with probabilities 0.79 and 0.16 for the 3 and 4 component models respectively. The sampling of the collapsed posterior as outlined in Algorithm (4) took less than 1 minute for 100,000 draws, thinned by 10. The resulting posterior mean actor positions are shown in 
Figure 1, where arrows represent directed ties between nodes and pie charts indicate the uncertainty in the cluster membership of actors.

Proposal variances for the Metropolis-Hastings moves were $\sigma_{z}^{2}=0.7$ for the latent actor positions and $\sigma_{\beta}^{2}=0.5$ for the intercept. Acceptance rates are displayed in Table 2, Figure 2 displays trace plots of the intercept $\beta$ and a sample actor position pre- and post-Procrustes matching demonstrating the effect of post processing the positions. The choice of hyperparameters, $\delta=0.103, \alpha=2$ and $\nu=3$, are in line with Handcock et al (2007). A Poisson(1) prior on the number of groups was used as per Nobile and Fearnside $(2007)$ and the prior variance of the cluster means were dependent on $\sigma_{g}^{2}$ and scaled by a factor of $\omega^{2}=10$. The analysis was reasonably insensitive to the choice of hyperparameters since the 3 cluster model chosen for a wide range of values of $\phi$. As described in Section 2, the priors are not identical for each model which must be considered when comparing inference using the collapsed sampling, inference using latentnet and the variational approach using VBLCPM.

Posterior model probabilities for the collapsed method are displayed in Table 1 together with approximate BIC values inferred using latentnet and VBLPCM. The lowest BIC value is the 'best' model. All approaches favoured 3 clusters in the network. Sampling the full posterior using latentnet took 24 minutes for 100,000 draws of the 1 to 5 component models compared to 1 minute for the collapsed sampling. There is further agreement in that the second lowest BIC values are for the 4 component model. The best estimate for the actor latent positions using latentnet for the 3 and 4 group models are displayed in Figure 3. The variational approach took 50 seconds to find the best of 10 modal variational estimates using VBLPCM (Salter-Townshend and Murphy 2012). The 3 and 4 cluster variational fits are displayed in Figure 4.
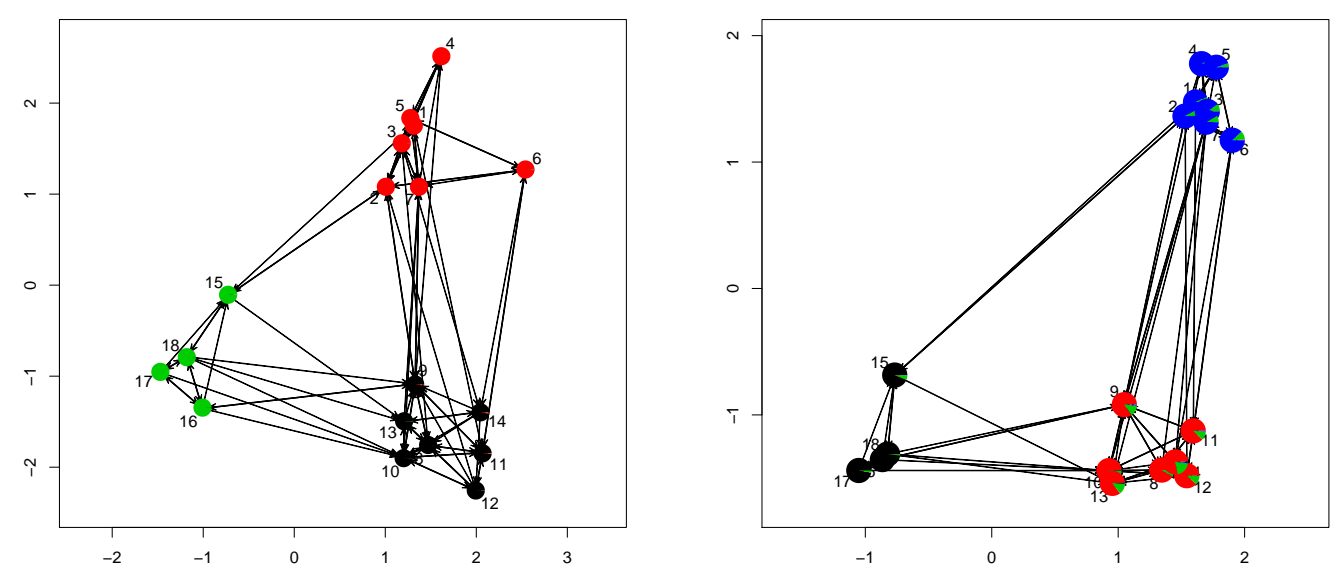

Figure 1: Sampson's monks posterior mean actor positions using the collapsed sampler for the most probable 3 and 4 group models (left and right hand plots respectively) with a pie chart depicting uncertainty of cluster memberships.

The results of our analysis for Sampson's monks network are qualitatively similar to the inference using latentnet. However inference using the collapsed model results in a dramatic reduction in CPU time. Qualitatively different results were seen for the variational approximation using VBLPCM with less separation of clusters and practically no uncertainty in cluster membership. Perhaps this is due to the approximation of the posterior distribution by the variational posterior. A major drawback of the 


\begin{tabular}{|l|c|c|c|c|c|}
\hline & $G=1$ & $G=2$ & $G=3$ & $G=4$ & $G=5$ \\
\hline Collapsed model probabilities $\pi(G \mid \mathbf{Y})$ & 0.0005 & 0.0092 & $\underline{0.7886}$ & 0.1604 & 0.1727 \\
latentnet BIC & 482.56 & 476.20 & $\underline{439.75}$ & 443.51 & 447.19 \\
VBLPCM BIC & 530.54 & 497.51 & $\underline{473.55}$ & 486.49 & 494.40 \\
\hline
\end{tabular}

Table 1: Posterior model probabilities for Sampson's monks for the collapsed sampling and BIC values fitting 5 models separately using latentnet and VBLPCM. (The model underlined denotes the best model for each method.)

\begin{tabular}{|l|c|}
\hline Update Type & Acceptance Rate (\%) \\
\hline Intercept $(\beta)$ & 25.53 \\
Latent Positions $(\mathbf{Z})$ & 23.64 \\
\hline Allocation Updates $(\mathbf{K})$ & Acceptance Rate (\%) \\
\hline Gibbs update & - \\
Move 1 & 1.89 \\
Move 2 & 15.61 \\
Move 3 & 1.62 \\
Ejection & 3.99 \\
Absorption & 3.99 \\
\hline
\end{tabular}

Table 2: Acceptance rates for collapsed model sampling for Sampson's monks.

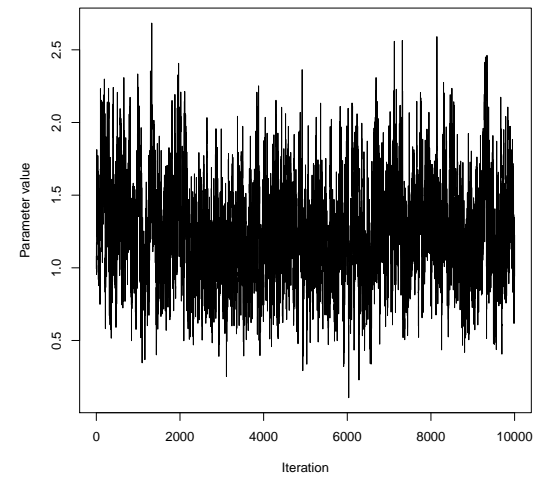

(a)

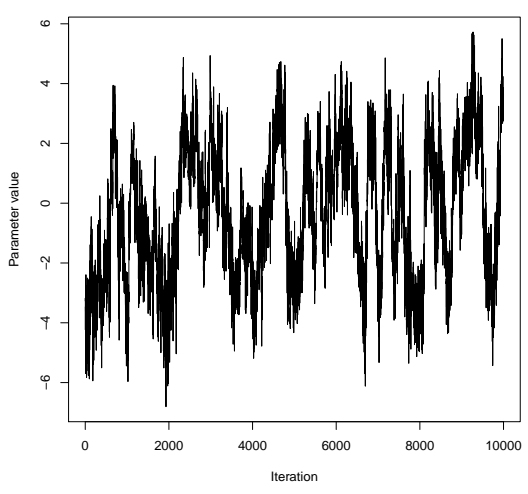

(b)

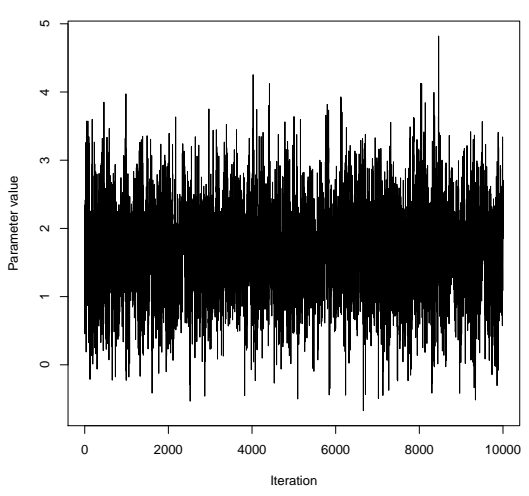

(c)

Figure 2: Trace plots of intercept $\beta$ (plot (a)) and one sample latent position $z_{(1,1)}$ pre- and postProcrustes matching (plots (b), (c) respectively) for Sampson's monks. 

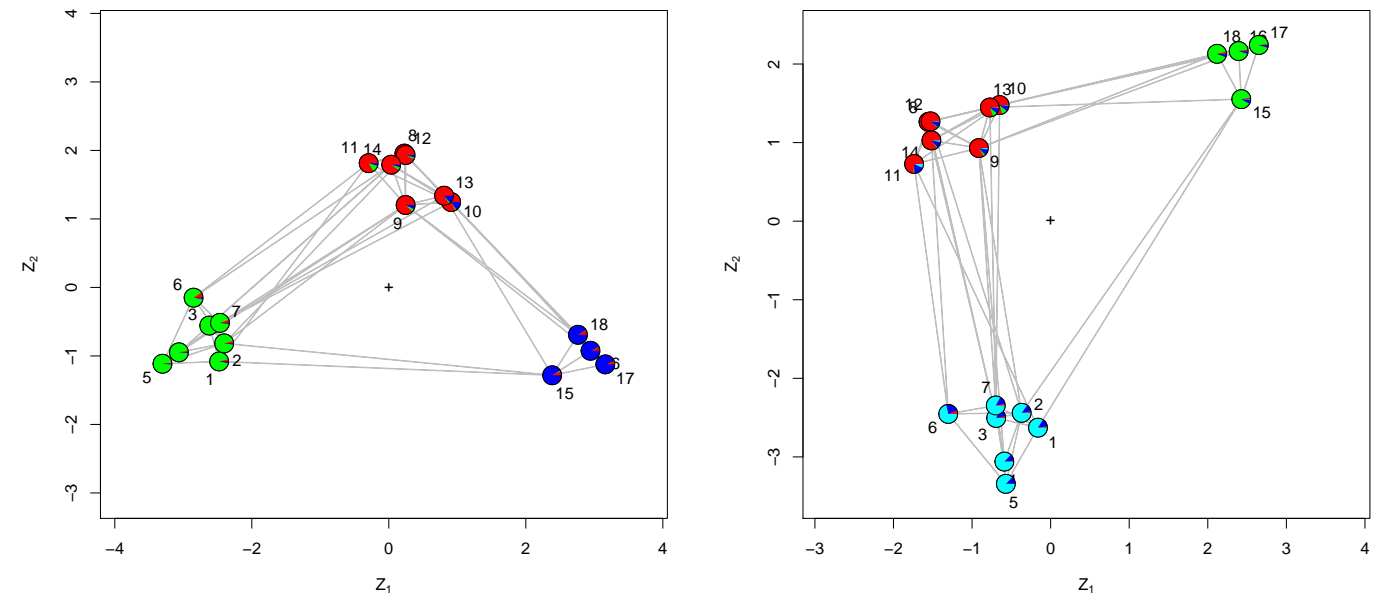

Figure 3: Posterior mean latent positions for a 3 and 4 component model for Sampson's monks using latentnet.
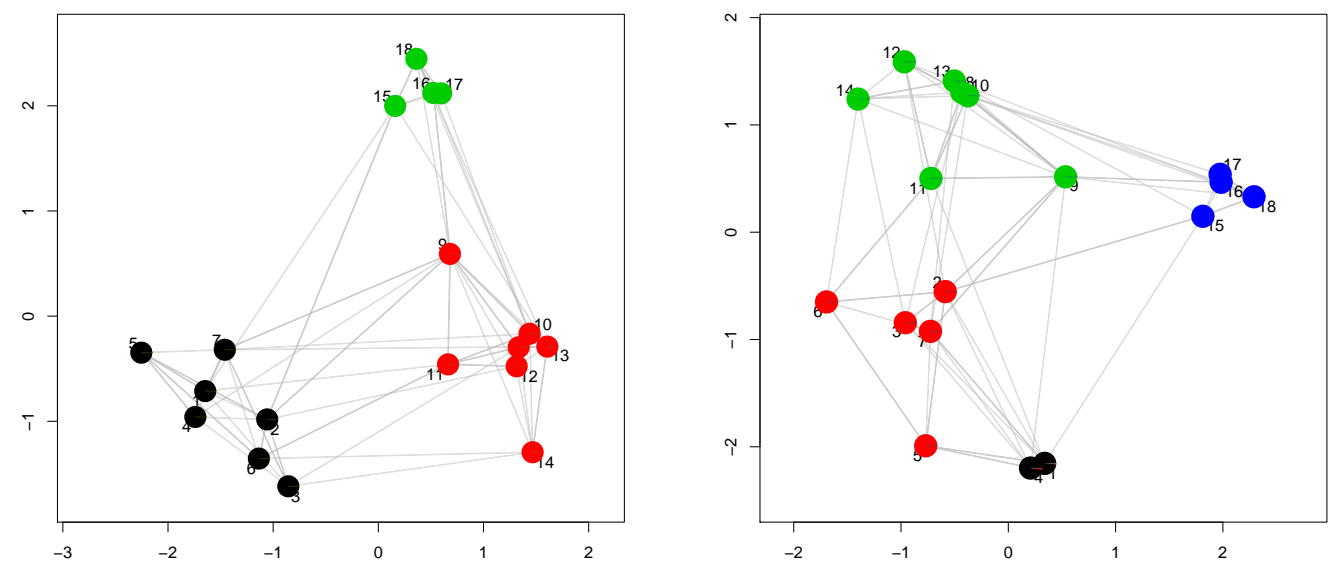

Figure 4: Variational Bayesian estimates of Latent positions for a 3 and 4 component model for Sampson's monks. 
variational approach is that the divergence between the two distributions can only be quantified up to an unknown constant of proportionality.

\subsection{Zachary's Karate Club}

Zachary's karate club (Zachary 1977) consists of 78 undirected friendship ties between 34 members of a karate club. The club split due to a disagreement between the club president and the coach, both of whom are included in the network as actors 1 and 34 respectively. The coach formed a new club with some of the members. It is interesting to compare the actual split of the club and the clustering of the friendship network. This is another example of a dynamic network which is usually examined in a static aggregated form in the social network analysis literature.

Using the collapsed inference, the 3 component model was favoured by a small margin with probability 0.38. Posterior model probabilities for the collapsed method and the approximate BIC inferred by latentnet and VBLPCM are displayed in Table 3. CPU time was 17 minutes for 1,000,000 samples drawn from the collapsed posterior, thinning by 100. The latent positions and the intercept mixed well (Figure 6) using proposal variances $\sigma_{z}^{2}=1.7$ and $\sigma_{\beta}^{2}=0.5$. However, moves 1 and 3 on the allocation vector were slower to mix than for the smaller monks network. Perhaps this is due to the increased size of the allocation vector. Thus the discrete set of possible allocation vectors to search across is very large. The same hyperparameters were used as in Section 5.1. The resulting posterior mean actor positions for the collapsed method are shown in Figure 5. There is good agreement between the actual club split and the clustering of our friendship network for the 2 group model. The only discrepancy is actor 9. He is clustered with the president in our analysis of this friendship network with probability 0.79. However in reality he stayed in the coach Mr Hi's karate club due to the fact that he was only three weeks away from a test for his black belt (master status) when the split in the club occurred (Zachary 1977). Otherwise there is good agreement. Combining two clusters of the 3 group model, mirrors the true split as before, again with the discrepancy of actor 9 . The 2 group model looks quite linear here perhaps suggesting that a one-dimensional latent space may be appropriate.

Inference using latentnet took 7 to 13 minutes to run 100,000 MCMC draws for each model. For up to maximum of 5 groups, the full inference took approximately 50 minutes. The latentnet position estimates are displayed in Figure 7. There is good cluster agreement between the 2 component model inferred using latentnet and the 2 component model inferred using the collapsed sampler. However, the 3 component model inferred by the collapsed sampler displays less uncertainty in group membership than the corresponding estimate using latentnet. Actor positions are qualitatively similar for the 2 component model but somewhat different for the 3 component model. A possible influence is the difference in priors used in the methods.

Using the variational Bayes package VBLPCM for 10 runs of up to 5 components took 7 minutes and favoured the 5 group model (Figure 8). It is very much a hard clustering with almost no uncertainty in actor allocations which may suggest problems with using Variational Bayesian methods on this network.

The results are qualitatively similar for the collapsed method and the latentnet 2 group model. Inference using the collapsed algorithm reduces computation time by at least a factor of 2 , compared to inference using latentnet, even though the collapsed sampler has used many more iterations than latentnet. The VBLPCM algorithm was faster but chose a 5 component model with practically no uncertainty in cluster membership. 



Figure 5: Zachary's karate club posterior mean actor positions using the collapsed sampler for the most probable 2 and 3 group models with a pie chart depicting uncertainty of cluster memberships.

\begin{tabular}{|l|c|c|c|c|c|}
\hline & $G=1$ & $G=2$ & $G=3$ & $G=4$ & $G=5$ \\
\hline Collapsed model probabilities $\pi(G \mid \mathbf{Y})$ & 0.2365 & 0.2807 & $\underline{0.3769}$ & 0.0885 & 0.0147 \\
latentnet BIC & 776.21 & $\underline{747.40}$ & 750.63 & 756.84 & 770.64 \\
VBLPCM BIC & 1267.67 & 1134.30 & 1109.42 & 1093.94 & $\underline{1092.48}$ \\
\hline
\end{tabular}

Table 3: Posterior model probabilities for Zachary's karate club data using the collapsed algorithm and BIC values using latentnet and VBLPCM. (The model underlined denotes the best model for each method.)

\begin{tabular}{|l|c|}
\hline Update Type & Acceptance Rate (\%) \\
\hline Intercept $(\beta)$ & 23.12 \\
Latent Positions $(\mathbf{Z})$ & 27.28 \\
\hline Allocation Updates $(\mathbf{K})$ & Acceptance Rate $(\%)$ \\
\hline Gibbs update & - \\
Move 1 & 0.66 \\
Move 2 & 7.50 \\
Move 3 & 0.54 \\
Ejection & 1.41 \\
Absorption & 2.23 \\
\hline
\end{tabular}

Table 4: Acceptance rates (\%) for Zachary's karate club. 


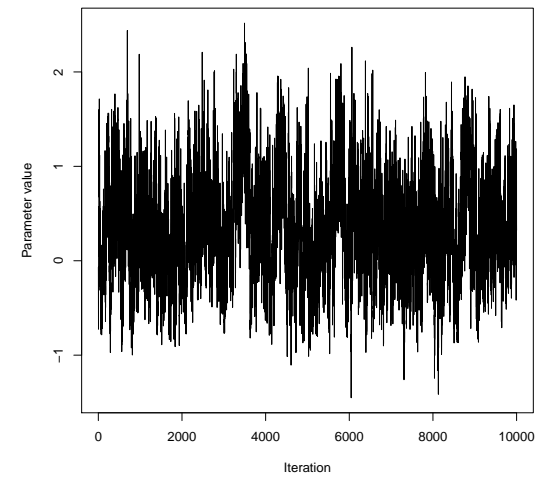

(a)

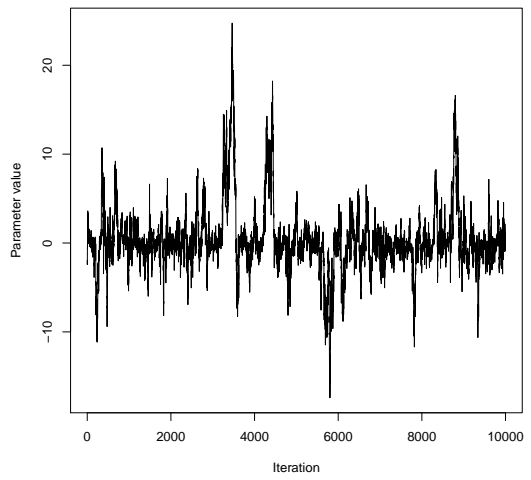

(b)

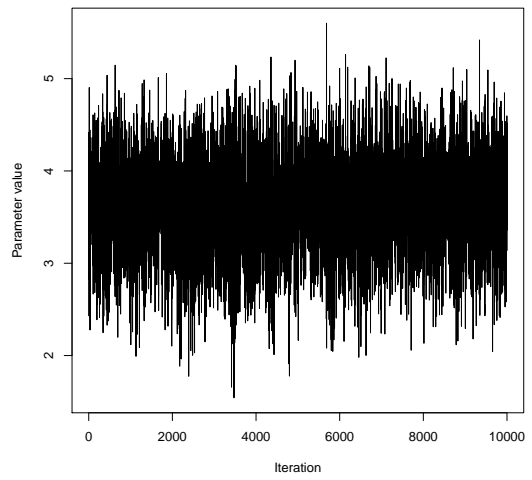

(c)

Figure 6: Trace plots of intercept $\beta$ (plot (a)) and one sample latent position $z_{(1,1)}$ pre- and postProcrustes matching (plots (b), (c) respectively) for Zachary's karate club.
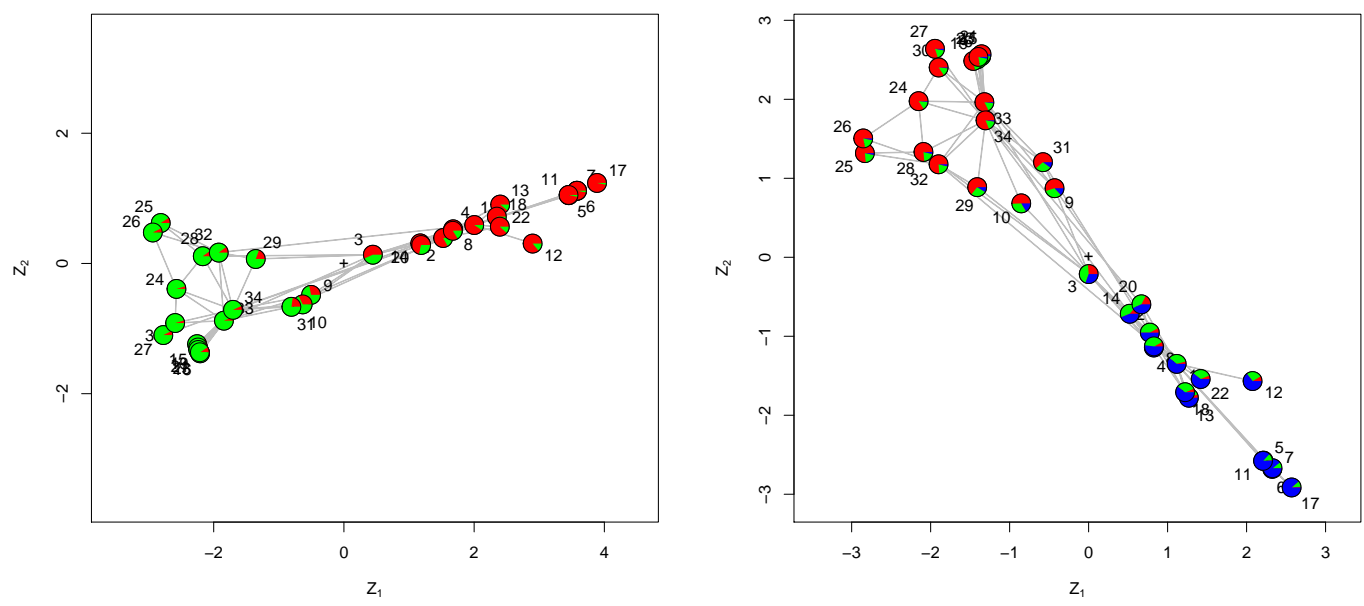

Figure 7: Posterior mean latent positions for a 2 and 3 cluster models for Zachary's karate club using latentnet. 

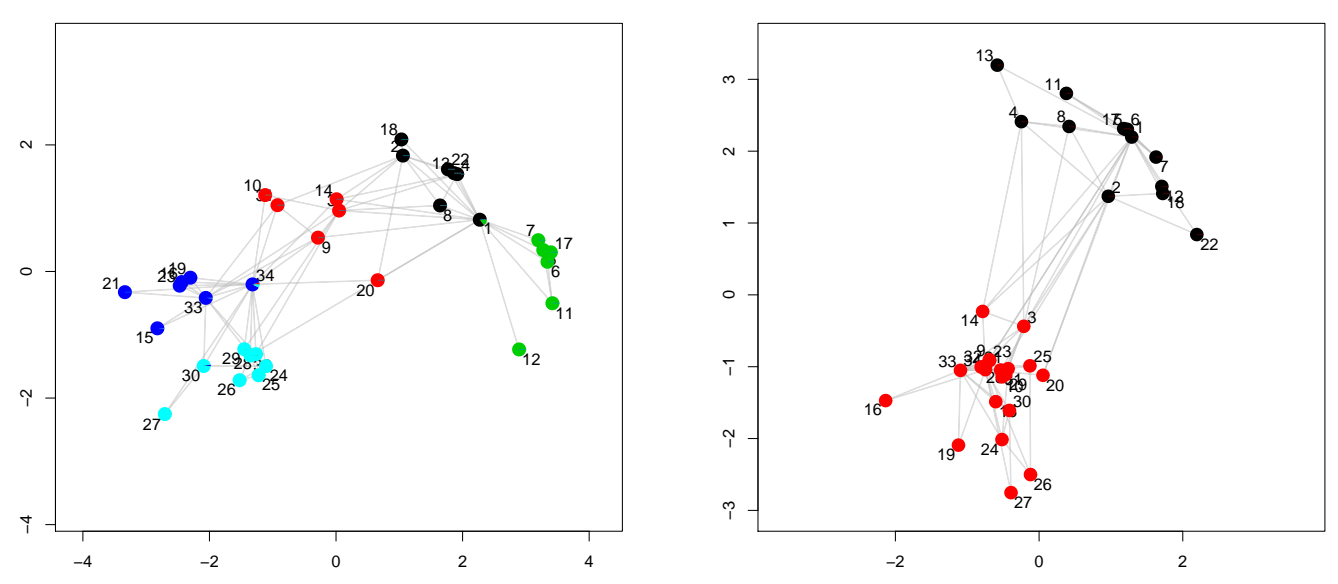

Figure 8: Variational Bayesian estimates of Latent positions of the 5 and 2 component model for Zachary's karate club.

\subsection{Dolphin Network}

The dolphin network studied by Lusseau et al (2003) represents social associations between 62 dolphins living off Doubtful Sound in New Zealand. It is an undirected graph with 159 ties.

The 2 group model was favoured by the collapsed allocation sampler and inference using latentnet. Posterior model probabilities for the collapsed method are displayed in Table 5 together with the inferred BIC approximations to the approximated model evidence using latentnet and VBLPCM. In total 1, 000,000 draws of the collapsed posterior took 48 minutes to run compared to 5 hours for latentnet with 100,000 MCMC draws of the posterior for 5 fitted models. Resulting positions and allocations are similar. The 5 group model was chosen using VBLPCM which took 40 minutes for 10 variational fits of up to 5 component models. The variational fit is displayed in Figure 12 and is very different from both latentnet (Figure 11) and our collapsed method (Figure 9).

Posterior mean actor positions inferred by the collapsed sampling are displayed in Figure 9. As before, prior hyperparameters were set to $\alpha=2, \delta=0.103, \nu=3$ and $\omega^{2}=10$. Good mixing can be seen in Figure 10 and acceptance rates are displayed in Table 6. The chain was thinned by 100. Proposal variances for the Metropolis-Hastings moves were $\sigma_{z}^{2}=3$ and $\sigma_{\beta}^{2}=0.2$ for the latent actor positions and for the intercept respectively.

Good agreement can be seen between inference using latentnet and the collapsed sampler choosing the 2 group model with qualitatively similar estimates of the latent actor positions as well as allocations. Results inferred by VBLPCM differed, favouring the 5 group model with very little uncertainty in group membership.

\section{Discussion}

A novel approach to model selection for the latent position cluster model for social networks has been presented. Integrating out most of the clustering parameters from the model analytically provides a fixed dimensional parameter space for trans-model inference, allowing joint inference on the number of clusters in the network. It avoids multiple approximations used by Handcock et al (2007) to estimate the model evidence, while simultaneously improving computational efficiency compared with standard 


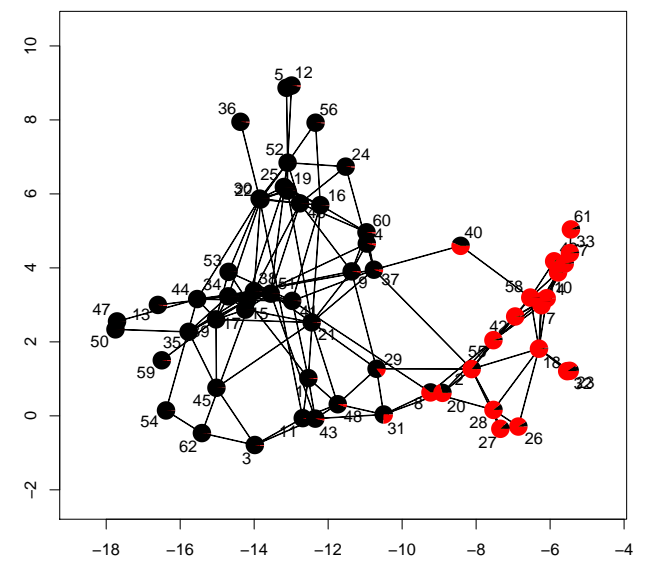

Figure 9: The dolphin network's posterior mean actor positions using the collapsed sampler for the most probable 2 group model with a pie chart depicting uncertainty of cluster memberships.

\begin{tabular}{|l|c|c|c|c|c|}
\hline & $G=1$ & $G=2$ & $G=3$ & $G=4$ & $G=5$ \\
\hline Collapsed model probabilities $\pi(G \mid \mathbf{Y})$ & 0.0394 & $\underline{0.8986}$ & 0.0583 & 0.0034 & 0.0003 \\
latentnet BIC & 1686.48 & $\underline{\underline{1660.16}}$ & 1667.23 & 1680.53 & 1690.32 \\
VBLPCM BIC & 3141.68 & $\underline{2537.09}$ & 2506.26 & 2449.28 & $\underline{2434.26}$ \\
\hline
\end{tabular}

Table 5: Posterior model probabilities for the dolphin network using the Collapsed algorithm and BIC using latentnet and VBLPCM (The model underlined denotes the best model for each method.)

\begin{tabular}{|l|c|}
\hline Update Type & Acceptance Rate (\%) \\
\hline Intercept $(\beta)$ & 26.33 \\
Latent Positions $(\mathbf{Z})$ & 27.37 \\
\hline Allocation Updates $(\mathbf{K})$ & Acceptance Rate (\%) \\
\hline Gibbs update & - \\
Move 1 & 0.04 \\
Move 2 & 2.73 \\
Move 3 & 0.09 \\
Ejection & 0.34 \\
Absorption & 0.38 \\
\hline
\end{tabular}

Table 6: Acceptance rates (\%) for the dolphin network. 


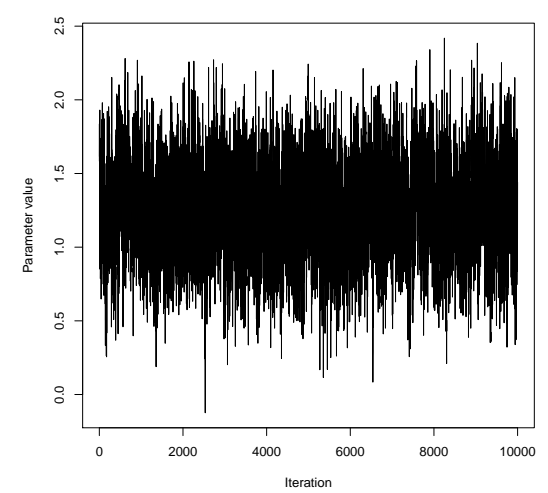

(a)

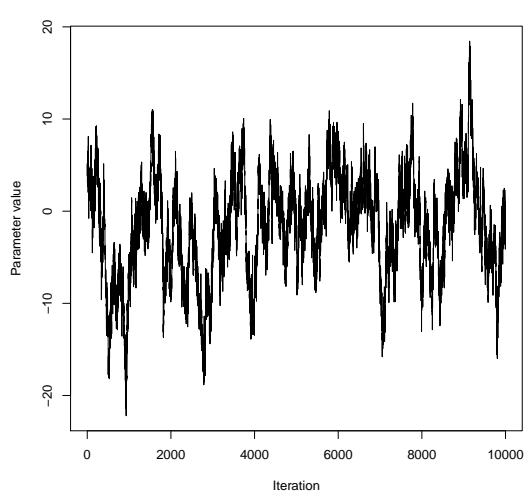

(b)

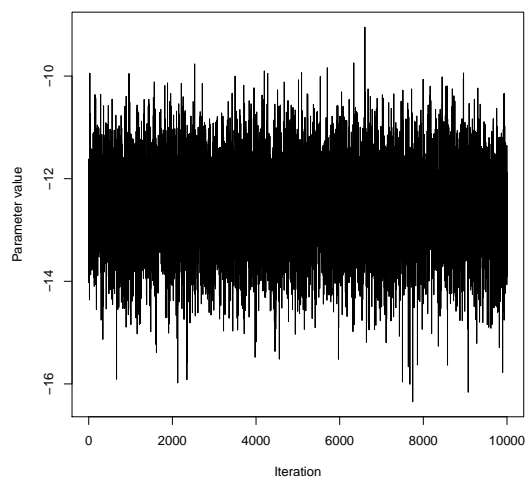

(c)

Figure 10: Trace plots of intercept $\beta$ (plot (a)) and one sample latent position $z_{(1,1)}$ pre- and postProcrustes matching (plots (b), (c) respectively) for the dolphin network.

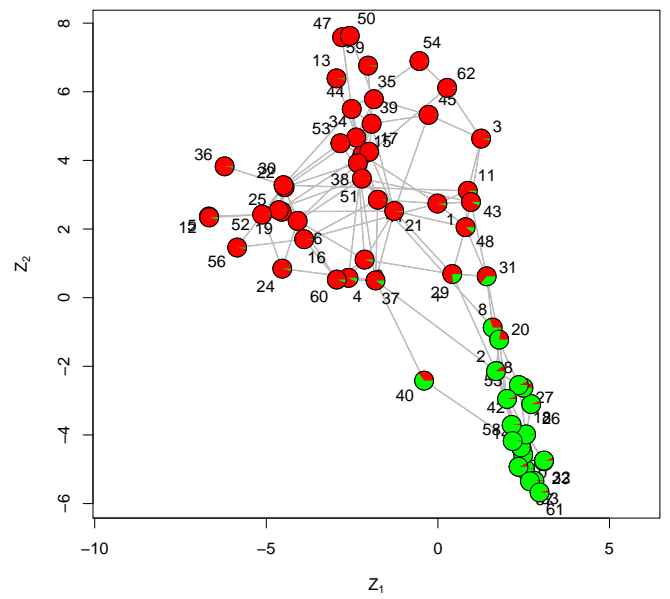

Figure 11: Posterior mean latentnet positions and uncertain clustering for the 2 component model of the dolphin network. 


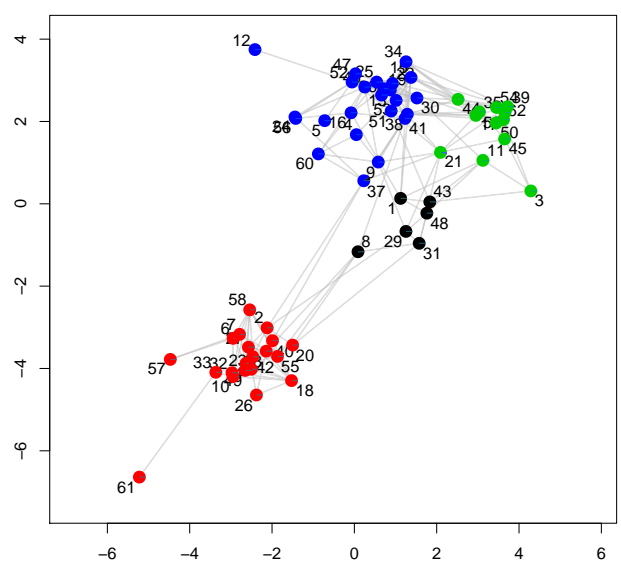

Figure 12: VBLPCM estimates of Latent positions for the 5 component model for the dolphin network.

methods. Parallelisation is possible for the likelihood, but not exploited in this paper and could give further decreases in computation time. Mixing can be poor for the allocation vector using the collapsed sampler but despite this runs faster than latentnet. On the other hand, collapsed sampling is coded in $C$ whereas latentnet uses $R$ and $C$. Analysis for latentnet visits all models in turn whereas the collapsed sampling is done in one chain.

Our methodology was demonstrated using three real data examples with comparisons to current methods. Similar results were found between our methods and sampling the full posterior for separate

models using latentnet. Substantial uncertainty in the number of clusters and cluster membership was evident.

\section{Appendix}

\subsection{Analytic integration of the clustering parameters from the latent po- sition cluster model}

The collapsing of the latent position cluster model is detailed here. The clustering parameters $\boldsymbol{\theta}=\left(\boldsymbol{\mu}, \boldsymbol{\tau}=1 / \boldsymbol{\sigma}^{2}, \boldsymbol{\lambda}\right)$ are integrated out of the model analytically. The collapsed posterior distribution 
can be written as

$$
\begin{aligned}
\pi(\mathbf{Z}, \beta, \mathbf{K}, G \mid \mathbf{Y})= & \int_{\boldsymbol{\tau}} \int_{\boldsymbol{\mu}} \int_{\boldsymbol{\lambda}} L(\mathbf{Y} \mid \mathbf{Z}, \beta) \pi(\mathbf{Z} \mid \boldsymbol{\mu}, \boldsymbol{\tau}, \mathbf{K}, G) \pi(\mathbf{K} \mid \boldsymbol{\lambda}, G) \pi(\boldsymbol{\lambda} \mid G) \pi(\boldsymbol{\mu} \mid \boldsymbol{\tau}, G) \\
& \pi(\boldsymbol{\tau} \mid G) \pi(\beta) \pi(G) \mathrm{d} \boldsymbol{\lambda} \mathrm{d} \boldsymbol{\mu} \mathrm{d} \boldsymbol{\tau} \\
= & L(\mathbf{Y} \mid \mathbf{Z}, \beta) \pi(\beta) \pi(G) \int_{\boldsymbol{\tau}} \pi(\boldsymbol{\tau} \mid G) \int_{\boldsymbol{\mu}} \pi(\mathbf{Z} \mid \boldsymbol{\mu}, \boldsymbol{\tau}, \mathbf{K}, G) \pi(\boldsymbol{\mu} \mid \boldsymbol{\tau}, G) \\
& \int_{\boldsymbol{\lambda}} \pi(\mathbf{K} \mid \boldsymbol{\lambda}, G) \pi(\boldsymbol{\lambda} \mid G) \mathrm{d} \boldsymbol{\lambda}, \mathrm{d} \boldsymbol{\mu} \mathrm{d} \boldsymbol{\tau} \\
= & L(\mathbf{Y} \mid \mathbf{Z}, \beta) \pi(\beta) \pi(G) \int_{\boldsymbol{\tau}} \prod_{g=1}^{G} \frac{\left(\frac{\delta}{2}\right)^{\frac{\alpha}{2}}}{\Gamma\left(\frac{\alpha}{2}\right)} \tau_{g}^{\frac{\alpha}{2}-1} \exp \left\{-\frac{\delta}{2} \tau_{g}\right\} \\
& \left.\int_{\boldsymbol{\mu}} \prod_{i=1}^{N} \prod_{g=1}^{G}\left({ }^{-2 \pi}\right)^{-d / 2} \exp \left\{-\frac{\tau_{g}}{2}\left(\mathbf{z}_{i}-\boldsymbol{\mu}_{g}\right)^{T} \mathbf{I}_{d}\left(\mathbf{z}_{i}-\boldsymbol{\mu}_{g}\right)\right\}\right)^{\mathbb{1}\left(k_{i}=g\right)} \\
& \prod_{g=1}^{G}\left(\frac{2 \pi \omega^{2}}{\tau_{g}}\right)^{-d / 2} \exp \left\{-\frac{\tau_{g}}{2 \omega^{2}}\left(\boldsymbol{\mu}_{g}^{T} \mathbf{I}_{d} \boldsymbol{\mu}_{g}\right)\right\} \\
& \int_{\boldsymbol{\lambda}} \prod_{g=1}^{G} \lambda_{g}^{n_{g}} \frac{\Gamma(G \nu)}{\Gamma(\nu)^{G}} \prod_{g=1}^{G} \lambda_{g}^{\nu-1} \mathrm{~d} \boldsymbol{\lambda}, \mathrm{d} \boldsymbol{\mu} \mathrm{d} \boldsymbol{\tau},
\end{aligned}
$$

where $n_{g}=\sum_{i=1}^{n} \mathbb{1}\left(k_{i}=g\right)$ and we can rearrange to get,

$$
\begin{aligned}
\pi(\mathbf{Z}, \beta, \mathbf{K}, G \mid \mathbf{Y})= & L(\mathbf{Y} \mid \mathbf{Z}, \beta) \pi(\beta) \pi(G) \frac{\Gamma(G \nu)}{\Gamma(\nu)^{G}}(2 \pi)^{-\frac{d}{2}(n+G)} \frac{\left(\frac{\delta}{2}\right)^{\frac{G \alpha}{2}}}{\Gamma\left(\frac{\alpha}{2}\right)^{G}}\left(\omega^{2}\right)^{-\frac{G d}{2}} \\
& \int_{\boldsymbol{\tau}} \prod_{g=1}^{G} \tau_{g}^{\frac{1}{2}\left(n_{g} d+d+\alpha-2\right)} \exp \left\{-\frac{\delta}{2} \tau_{g}\right\} \\
& \int_{\boldsymbol{\mu}} \prod_{g=1}^{G} \exp \left\{-\frac{\tau_{g}}{2}\left(\sum_{i: k_{i}=g}\left\|\mathbf{z}_{i}-\boldsymbol{\mu}_{g}\right\|^{2}+\frac{1}{\omega^{2}}\left\|\boldsymbol{\mu}_{g}\right\|^{2}\right)\right\} \\
& \int_{\boldsymbol{\lambda}} \prod_{g=1}^{G} \lambda_{g}^{n_{g}+\nu-1} \mathrm{~d} \boldsymbol{\lambda} \mathrm{d} \boldsymbol{\mu} \mathrm{d} \boldsymbol{\tau} .
\end{aligned}
$$

The mixing weights $\boldsymbol{\lambda}$ are collapsed or integrated out of this expression using the Dirichlet density where,

$$
\int_{\boldsymbol{\lambda}} \prod_{g=1}^{G} \lambda_{g}^{n_{g}+\nu-1} \mathrm{~d} \boldsymbol{\lambda}=\frac{\prod_{g=1}^{G} \Gamma\left(n_{g}+\nu\right)}{\Gamma\left(\sum_{g=1}^{G}\left(n_{g}+\nu\right)\right)}=\frac{\prod_{g=1}^{G} \Gamma\left(n_{g}+\nu\right)}{\Gamma(n+G \nu)} .
$$


The cluster means $\boldsymbol{\mu}$ are collapsed using Multivariate Normal densities, with mean $\frac{\sum_{i: k_{i}=g} \mathbf{z}_{i}}{\left(n_{g}+\frac{1}{\omega^{2}}\right)}$ and covariance matrix $\left(\tau_{g}\left(n_{g}+\frac{1}{\omega^{2}}\right) \mathbf{I}\right)^{-1}$,

$$
\begin{aligned}
\int_{\boldsymbol{\mu}_{g=1}} \prod_{g=1}^{G} \exp & \left\{-\frac{\tau_{g}}{2}\left(\sum_{i: k_{i}=g}\left\|\mathbf{z}_{i}-\boldsymbol{\mu}_{g}\right\|^{2}+\frac{1}{\omega^{2}}\left\|\boldsymbol{\mu}_{g}\right\|^{2}\right)\right\} \\
& =\int_{\boldsymbol{\mu}_{g=1}} \prod_{g=1}^{\exp }\left\{-\frac{\tau_{g}}{2}\left(\sum_{i: k_{i}=g}\left(\mathbf{z}_{i}^{T} \mathbf{z}_{i}-2 \mathbf{z}_{i}^{T} \boldsymbol{\mu}_{g}+\boldsymbol{\mu}_{g}^{T} \boldsymbol{\mu}_{g}\right)+\frac{1}{\omega^{2}} \boldsymbol{\mu}_{g}^{T} \boldsymbol{\mu}_{g}\right)\right\} \mathrm{d} \boldsymbol{\mu} \\
& =\int_{\boldsymbol{\mu}_{g=1}} \prod_{g=1}^{G} \exp \left\{-\frac{\tau_{g}}{2}\left(\boldsymbol{\mu}_{g}^{T} \boldsymbol{\mu}_{g}\left(n_{g}+\frac{1}{\omega^{2}}\right)-2 \sum_{i: k_{i}=g} \mathbf{z}_{i}^{T} \boldsymbol{\mu}_{g}+\sum_{i: k_{i}=g} \mathbf{z}_{i}^{T} \mathbf{z}_{i}\right)\right\} \mathrm{d} \boldsymbol{\mu} \\
& =\int_{\boldsymbol{\mu}_{g=1}} \prod_{g=1}^{G} \exp \left\{-\frac{\tau_{g}}{2}\left(\left(n_{g}+\frac{1}{\omega^{2}}\right)\left\|\boldsymbol{\mu}_{g}-\frac{\sum_{i: k_{i}=g} \mathbf{z}_{i}}{\left(n_{g}+\frac{1}{\omega^{2}}\right)}\right\|^{2}-\frac{\left\|\sum_{i: k_{i}=g} \mathbf{z}_{i}\right\|^{2}}{\left(n_{g}+\frac{1}{\omega^{2}}\right)}+\sum_{i: k_{i}=g}\left\|\mathbf{z}_{i}\right\|^{2}\right)\right\} \mathrm{d} \boldsymbol{\mu} \\
& =\prod_{g=1}^{G} 2 \pi^{d / 2}\left(\tau_{g}\left(n_{g}+\frac{1}{\omega^{2}}\right)\right)^{-\frac{d}{2}} \exp \left\{-\frac{\tau_{g}}{2}\left(\sum_{i: k_{i}=g}\left\|\mathbf{z}_{i}\right\|^{2}-\frac{\left\|\sum_{i: k_{i}=g} \mathbf{z}_{i}\right\|^{2}}{\left(n_{g}+\frac{1}{\omega^{2}}\right)}\right)\right\} .
\end{aligned}
$$

Having integrated out $\boldsymbol{\mu}$ and $\boldsymbol{\lambda}$ analytically, the resulting collapsed posterior is

$$
\begin{aligned}
\pi(\mathbf{Z}, \beta, \mathbf{K}, G \mid \mathbf{Y}) & =L(\mathbf{Y} \mid \mathbf{Z}, \beta) \pi(\beta) \pi(G) \frac{\Gamma(G \nu)}{\Gamma(\nu)^{G}}(2 \pi)^{-\frac{d}{2}(n+G)} \frac{\left(\frac{\delta}{2}\right)^{\frac{G \alpha}{2}}}{\Gamma\left(\frac{\alpha}{2}\right)^{G}}\left(\omega^{2}\right)^{-\frac{G d}{2}}(2 \pi)^{\frac{G d}{2}} \prod_{g=1}^{G} \frac{\Gamma\left(n_{g}+\nu\right)}{\Gamma(n+G \nu)} \\
& \times \int_{\boldsymbol{\tau}} \prod_{g=1}^{G}\left(n_{g}+\frac{1}{\omega^{2}}\right)^{-\frac{d}{2}} \tau_{g}^{\frac{1}{2}\left(d n_{g}+\alpha-2\right)} \exp \left\{-\frac{\tau_{g}}{2}\left(\delta+\sum_{i: k_{i}=g}\left\|\mathbf{z}_{i}\right\|^{2}-\frac{\left\|\sum_{i: k_{i}=g} \mathbf{z}_{i}\right\|^{2}}{\left(n_{g}+\frac{1}{\omega^{2}}\right)}\right)\right\} \mathrm{d} \tau .
\end{aligned}
$$

The cluster precision parameter $\tau_{g}=1 / \sigma_{g}^{2}$ is collapsed using $\operatorname{Gamma}\left(\alpha^{\prime}, \beta^{\prime}\right)$ densities, where $\alpha^{\prime}=\frac{n_{g} d+\alpha}{2}$ and $\beta^{\prime}=\frac{1}{2}\left(\sigma+\sum_{i: k_{i}=g}\left\|\mathbf{z}_{i}\right\|^{2}-\frac{\left\|\sum_{i: k_{i}=g} \mathbf{z}_{i}\right\|^{2}}{\left(n_{g}+\frac{1}{\omega^{2}}\right)}\right)$, as follows,

$$
\begin{aligned}
\pi(\mathbf{Z}, \beta, \mathbf{K}, G \mid \mathbf{Y}) & =L(\mathbf{Y} \mid \mathbf{Z}, \beta) \pi(\beta) \pi(G) \frac{\Gamma(G \nu)}{\Gamma(\nu)^{G}}(2 \pi)^{-\frac{d}{2}(n+G)} \frac{\left(\frac{\delta}{2}\right)^{\frac{G \alpha}{2}}}{\Gamma\left(\frac{\alpha}{2}\right)^{G}}\left(\omega^{2}\right)^{-\frac{G d}{2}}(2 \pi)^{\frac{G d}{2}} \prod_{g=1}^{G} \frac{\Gamma\left(n_{g}+\nu\right)}{\Gamma(n+G \nu)} \\
& \times \prod_{g=1}^{G}\left(n_{g}+\frac{1}{\omega^{2}}\right)^{-\frac{d}{2}} \Gamma\left(\frac{n_{g} d+\alpha}{2}\right)\left(\frac{1}{2}\left(\delta+\sum_{i: k_{i}=g}\left\|\mathbf{z}_{i}\right\|^{2}-\frac{\left\|\sum_{i: k_{i}=g} \mathbf{z}_{i}\right\|^{2}}{\left(n_{g}+\frac{1}{\omega^{2}}\right)}\right)\right)^{-\left(\frac{n_{g} d+\alpha}{2}\right)} .
\end{aligned}
$$


Finally, the fully collapsed posterior for the latent position cluster model, including expressions for the likelihood, the prior for $\beta$ and the prior on for the number of groups $G$ is

$$
\begin{aligned}
\pi(\mathbf{Z}, \beta, \mathbf{K}, G \mid \mathbf{Y})= & \prod_{i=1}^{n} \prod_{j \neq i} \frac{\exp \left\{y_{i j}\left(\beta-\left\|\mathbf{z}_{i}-\mathbf{z}_{j}\right\|\right)\right\}}{1+\exp \left(\beta-\left\|\mathbf{z}_{i}-\mathbf{z}_{j}\right\|\right)} \\
& \times \frac{1}{\sqrt{2 \pi \psi}} \exp \left\{-\frac{(\beta-\xi)^{2}}{2 \psi}\right\} \frac{\exp \{-1\}}{G !} \\
& \times \frac{\Gamma(G \nu)}{\Gamma(\nu)^{G}} \pi^{-\frac{d n}{2}} \frac{(\delta)^{\frac{G \alpha}{2}}}{\Gamma\left(\frac{\alpha}{2}\right)^{G}}\left(\omega^{2}\right)^{-\frac{G d}{2}} \frac{\prod_{g=1}^{G} \Gamma\left(n_{g}+\nu\right)}{\Gamma(n+G \nu)} \\
& \times \prod_{g=1}^{G} \frac{\Gamma\left(\frac{n_{g} d+\alpha}{2}\right)}{\left(n_{g}+\frac{1}{\omega^{2}}\right)^{\frac{d}{2}}}\left(\delta+\sum_{i: k_{i}=g}\left\|\mathbf{z}_{i}\right\|^{2}-\frac{\left\|\sum_{i: k_{i}=g} \mathbf{z}_{i}\right\|^{2}}{\left(n_{g}+\frac{1}{\omega^{2}}\right)}\right)^{-\left(\frac{n_{g} d+\alpha}{2}\right)} .
\end{aligned}
$$

Acknowledgements: Nial Friel and Caitríona Ryan's research was supported by a Science Foundation Ireland Research Frontiers Program grant, 09/RFP/MTH2199. This research was also supported in part by a research grant from Science Foundation Ireland (SFI) under Grant Number SFI/12/RC/2289. Jason Wyse"s research was supported through the STATICA project, a Principal Investigator program of Science Foundation Ireland, 08/IN.1/I1879.

\section{Supplemental Materials}

C code: The supplemental files for this article include the $\mathrm{C}$ implementation of the algorithms of Section 4. The examples of Section 5 can be reproduced by calling an $\mathrm{R}$ script also provided. Please see the file README contained within the accompanying tar file for more details.

\section{References}

Adamic, L., R. Lukose, A. Puniyani and B. Huberman (2001), Search in power-law networks. Physical Review E 64(4), 046135

Breslow, N. (1996), Statistics in epidemiology: the case-control study. Journal of the American Statistical Association 91(433), 14-28

Carpaneto, G. and P. Toth (1980), Algorithm 548: Solution of the assignment problem [H]. ACM Transactions on Mathematical Software (TOMS) 6(1), 104-111

Dellaportas, P. and I. Papageorgiou (2006), Multivariate mixtures of normals with unknown number of components. Statistics and Computing 16(1), 57-68

Faloutsos, M., P. Faloutsos and C. Faloutsos (1999), On power-law relationships of the internet topology. In ACM SIGCOMM Computer Communication Review, vol. 29, pp. 251-262, ACM

Fraley, C. and A. E. Raftery (2002), Model-based clustering, discriminant analysis, and density estimation. Journal of the American Statistical Association 97(458), 611-631

Fraley, C. and A. E. Raftery (2003), Enhanced model-based clustering, density estimation, and discriminant analysis software: MCLUST. Journal of Classification 20(2), 263-286

Friel, N. and J. Wyse (2012), Estimating the evidence-a review. Statistica Neerlandica 66(3), 288-308 
Handcock, M., A. Raftery and J. Tantrum (2007), Model-based clustering for social networks. Journal of the Royal Statistical Society: Series A (Statistics in Society) 170(2), 301-354

Hoff, P.D.and Raftery, A. and M. Handcock (2002), Latent space approaches to social network analysis. Journal of the American Statistical Association 97(460), 1090-1098

Kolaczyk, E. (2009), Statistical analysis of network data: methods and models. Springer

Krivitsky, P. and M. Handcock (2008), Fitting Latent Cluster Models for Networks with latentnet. Journal of Statistical Software 24(5), 1-23

Krivitsky, P. N. and M. S. Handcock (2013), latentnet: Latent position and cluster models for statistical networks. The Statnet Project (http://www.statnet.org), R package version 2.4-4

Lusseau, D., K. Schneider, O. Boisseau, P. Haase, E. Slooten and S. Dawson (2003), The bottlenose dolphin community of Doubtful Sound features a large proportion of long-lasting associations. Behavioral Ecology and Sociobiology 54(4), 396-405

Michailidis, G. (2012), Statistical Challenges in Biological Networks. Journal of Computational and Graphical Statistics 21(4), 840-855

Nobile, A. (2007), Bayesian finite mixtures: a note on prior specification and posterior computation. arXiv preprint arXiv:0711.0458

Nobile, A. and A. Fearnside (2007), Bayesian finite mixtures with an unknown number of components: the allocation sampler. Statistics and Computing 17(2), 147-162

Nowicki, K. and T. Snijders (2001), Estimation and prediction for stochastic blockstructures. Journal of the American Statistical Association 96(455), 1077-1087

Phillips, D. and A. Smith (1996), Bayesian model comparison via jump diffusions. Markov chain Monte Carlo in practice pp. 215-239

Raftery, A., X. Niu, P. Hoff and K. Yeung (2012), Fast inference for the latent space network model using a case-control approximate likelihood. Journal of Computational and Graphical Statistics 21(4), 901-919

Richardson, S. and P. Green (1997), On Bayesian Analysis of Mixtures with an Unknown Number of Components (with discussion). Journal of the Royal Statistical Society: Series B (Statistical Methodology) 59, 731-792

Robins, G., T. Snijders, P. Wang, M. Handcock and P. Pattison (2007), Recent developments in exponential random graph (p*) models for social networks. Social Networks 29(2), 192-215

Salter-Townshend, M. and T. Murphy (2012), Variational Bayesian Inference for the Latent Position Cluster Model for network data. Computational Statistics $\&$ Data Analysis 57(1), 661-671

Sampson, S. (1968), A novitiate in a period of change: An experimental and case study of social relationships. Ph.D. thesis, Cornell University, September

Schwarz, G. (1978), Estimating the dimension of a model. The Annals of Statistics 6(2), 461-464

Shortreed, S., M. Handcock and P. Hoff (2006), Positional estimation within a latent space model for networks. Methodology: European Journal of Research Methods for the Behavioral and Social Sciences 2(1), 24-33

Sibson, R. (1978), Studies in the robustness of multidimensional scaling: Procrustes statistics. Journal of the Royal Statistical Society. Series B (Methodological) pp. 234-238 
Stephens, M. (2000), Bayesian analysis of mixture models with an unknown number of components-an alternative to reversible jump methods. Annals of Statistics pp. 40-74

Wasserman, S. and J. Galaskiewicz (1994), Advances in social network analysis: Research in the social and behavioral sciences. Sage Publications, Incorporated

Wasserman, S. and P. Pattison (1996), Logit models and logistic regressions for social networks: I. An introduction to Markov graphs and p*. Psychometrika 61(3), 401-425

Wyse, J. and N. Friel (2012), Block clustering with collapsed latent block models. Statistics and Computing 22(2), 415-428

Zachary, W. (1977), An information flow model for conflict and fission in small groups. Journal of anthropological research pp. 452-473 